\title{
EFICIENCIA DE UN SEDIMENTADOR LAMINAR Y CONVENCIONAL PARA ELIMINAR SÓLIDOS
}

\author{
Tania E. Guerrero Vejarano ${ }^{1 *}$, Juan C. Salazar Álvarez², José L. Paredes Salazar³
}

\begin{abstract}
RESUMEN
El presente trabajo de investigación tuvo como objetivo determinar la eficiencia del sedimentador laminar y convencional para eliminar diferentes concentraciones de sólidos en suspensión, para ello se tomaron en cuenta diferentes parámetros fisicoquímicos (temperatura, $\mathrm{pH}$, turbiedad, oxígeno disuelto y residuo seco).

Para determinar los parámetros fisicoquímicos se prepararon muestras de agua a diferentes concentraciones $(1,2$ y $3 \%$ ) de sólidos, las cuales se analizaron a la entrada y salida de los sedimentadores, en tratamientos con coagulante y sin coagulante, con tres repeticiones a cada tratamiento. Para determinar cuál de los sedimentadores fue más eficiente, se realizó el análisis de varianza y prueba de Tukey de los factores evaluados (tipo de sedimentador, coagulante y concentración de sólidos), obteniéndose como resultado diferencias significativas entre ambos sedimentadores con respecto al tipo de concentración y según el tratamiento con coagulante o sin coagulante, llegando a la conclusión que el sedimentador laminar tiene una eficiencia de $93,85 \%$, siendo más eficiente que el convencional con una eficiencia de 78,00\%, en la concentración del $2 \%$ de sólidos utilizando coagulante.
\end{abstract}

Palabras clave: sedimentación, eficiencia, sólidos sedimentables, floculación, sedimentador laminar, sedimentador convencional

\begin{abstract}
The objective of this research was to determine the efficiency of the laminar and conventional sedimentation system to eliminate different concentrations of suspended solids, for which different physicochemical parameters were taken into account (temperature, $\mathrm{pH}$, turbidity, dissolved oxygen and dry residue).

To determine the physicochemical parameters, water samples were prepared at different concentrations (1,2 and 3\%) of solids, which were analyzed at the inlet and outlet of the settler, in treatments with coagulant and without coagulant, with three repetitions at each treatment. To determine which of the sedimentation was more efficient, analysis of variance and Tukey test of the evaluated factors (type of settler, coagulant and concentration of solids) were performed, obtaining as a result significant differences between both settlings with

\footnotetext{
${ }^{1}$ Universidad Nacional Agraria de la Selva - Carretera Centra km 1,21 Tingo María, Perú. tania.guerrero@unas. edu.pe

${ }^{2}$ CONSEGESA S.A.

${ }^{3}$ Universidad Nacional Agraria de la Selva
} 
respect to the type of concentration and according to the treatment with coagulant or without coagulant, reaching the conclusion that the laminar settler has an efficiency of $93.85 \%$, being more efficient than the conventional one with an efficiency of $78.00 \%$, in the concentration of $2 \%$ of solids using coagulant.

Key words: sedimentation, efficiency, sedimentable solids, flocculation, laminar sedimentation, conventional sedimentation

\section{INTRODUCCIÓN}

La contaminación del agua por sólidos disueltos y en suspensión es el problema más común que afecta a las aguas residuales. El agua es importante para la salud humana, la purificación del agua es esencial para conservarla. El tratamiento de aguas residuales consiste en una serie de procesos físicos, químicos y biológicos que tienen como fin eliminar los contaminantes físicos, químicos y biológicos presentes en el agua provenientes del uso humano, esta situación se ha mejorado utilizando tratamientos básicos, entre ellos sedimentación y floculación, donde la calidad del efluente referido a los sólidos en suspensión o la turbidez depende del diseño de los sedimentadores ${ }^{1}$. Se evidencian estudios en la velocidad de sedimentación en flóculos de aluminio y de hierro con una gravedad específica de aproximadamente 1,002, un tamaño de partícula tan grande como $1 \mathrm{~mm}$ y una velocidad de sedimentación a $10^{\circ} \mathrm{C}$ de aproximadamente $0,8 \mathrm{~mm} / \mathrm{s}$, el tiempo de sedimentación de diversos tipos de floculantes como las sales de aluminio y férrico oscila entre 10 y $40 \mathrm{~min}^{2}$, esto depende de muchos factores: tipo de impureza, temperatura y $\mathrm{pH}$. La turbiedad es originada por las partículas en suspensión o coloides (arcillas, limo, tierra finamente dividida, etc.). Elevados niveles de turbidez pueden proteger a los microorganismos de los efectos de la desinfección, estimular la proliferación de bacterias y aumentar la demanda de cloro ${ }^{3}$. Los procesos que más afectan la eficiencia de los sedimentadores en la remoción de la turbidez del fluido y en la calidad del agua obtenida es el proceso de coagulación y floculación, ya que una adecuada selección del coagulante y una eficiente dosificación del mismo, genera una mayor cantidad de formación de partículas grandes en el floculador para posteriormente ser eliminadas en el sedimentador. ${ }^{4}$. También hay reportes sobre diseño de sedimentadores, sin embargo no hay reportes sobre la comparación de la eficiencia de sedimentadores que son ampliamente utilizados en los procesos de tratamiento, hay diversos tipos de sedimentadores y clarificadores y no hay evidencias sobre la eficiencia en los procesos de remoción de sólidos disueltos y en suspensión, es precisamente donde se enfoca el presente estudio en determinar la eficiencia del sedimentador laminar y convencional para eliminar diferentes concentraciones de sólidos en suspensión. 


\section{PARTE EXPERIMENTAL}

Se utilizó dos sedimentadores (convencional y laminar) diseñados y construidos según las especificaciones de la CEPIS. Simuló agua de río, se preparó en el laboratorio de tratamiento de aguas residuales, agua con tierra colectada del Bosque Reservado de la UNAS. Se utilizó como coagulante sulfato de aluminio y para regular el $\mathrm{pH}$ hidróxido de calcio. Para determinar la turbiedad se usó cloruro férrico, se adquirió de Merck. Asimismo, se usó una balanza analítica modelo Te214s-0ce de SARTORIUS, termómetro Lanceta HG, peachimetro de mesa marca ATC, espectrofotómetro UV Visible Thermo Scientific Modelo 2000, estufa MEMMERT modelo Be 20.

\section{METODOLOGÍA}

Diseño de los sedimentadores: La metodología aplicada es de la CEPIS5

Diseño del sedimentador convencional (CEPIS/OPS), Para el diseño del sedimentador convencional, se tuvo que trabajar con caudales constantes.

a. Zona de sedimentación

- Volumen del sedimentador (V): $50 \mathrm{~L}=0,05 \mathrm{~m} 3$, Calculamos el caudal (Q)

$$
\begin{gathered}
V=Q \times T_{0} \\
Q=\frac{V}{T_{0}}
\end{gathered}
$$

Donde:

$$
\begin{aligned}
& \mathrm{V}=\text { Volumen del sedimentador }\left(\mathrm{m}^{3}\right) \\
& \mathrm{Q}=\text { Caudal }\left(\mathrm{m}^{3} / \mathrm{s}\right) \\
& \mathrm{T}_{0}=\text { Tiempo de retención }(\mathrm{s}) \\
& \mathrm{Q}=\frac{0,05 \mathrm{~m}^{3} \times 1 \mathrm{~h}}{1,5 \mathrm{~h} \times 3600 \mathrm{~s}}=9,25 \times 10^{-6} \frac{\mathrm{m}^{3}}{\mathrm{~s}} \times \frac{1000 \mathrm{~L}}{1 \mathrm{~m}^{3}} \times \frac{1000 \mathrm{~mL}}{1 \mathrm{~L}} \\
& \mathrm{Q}=9,25 \mathrm{~mL} / \mathrm{s}
\end{aligned}
$$

- Área del sedimentador (A):

Se deberá cumplir con la siguiente relación:

$$
\begin{aligned}
& \mathrm{L}=6 \times \mathrm{H} \\
& 5<\frac{\mathrm{L}}{\mathrm{H}}<25 \\
& 2<\frac{\mathrm{L}}{\mathrm{B}}<5
\end{aligned}
$$

Por tanto:

$$
\mathrm{A}=\frac{\mathrm{V}}{\mathrm{H}}
$$


Donde:

$$
\begin{aligned}
& \mathrm{L}=\text { Largo }(\mathrm{m}) \\
& \mathrm{H}=\text { Profundidad }(\mathrm{m}) \\
& \mathrm{B}=\text { Ancho }(\mathrm{m}) \\
& \mathrm{A}=\text { Área }\left(\mathrm{m}^{2}\right) \\
& \qquad A=\frac{0,05 \mathrm{~m}^{3}}{0,18 \mathrm{~m}} \\
& \qquad A=0,2777 \mathrm{~m}^{2}
\end{aligned}
$$

De (1)

$$
\text { Si: } \mathrm{H}=0,18 \mathrm{~m}
$$

Por lo tanto:

$$
\mathrm{L}=6 \times 0,18
$$$$
\mathrm{L}=1,08 \mathrm{~m}
$$

De (2)

$$
5<\frac{L}{H}<25
$$

$5<\frac{1,08}{0,18}<25$

$$
\begin{gathered}
\frac{1,08}{0,18}=6 \\
B=\frac{\text { Área }}{L}=\frac{0,2777 \mathrm{~m}^{2}}{1,08 \mathrm{~m}}=0,2572 \mathrm{~m}
\end{gathered}
$$

De (3) $\quad 2<\frac{1,08}{0,2572}<5$

$$
\frac{1,08}{0,2572}=4,1999
$$

Las dimensiones del sedimentador son:

$$
\begin{aligned}
& \mathrm{V}=0,05 \mathrm{~m}^{3} \\
& \mathrm{~L}=1,08 \mathrm{~m} \\
& \mathrm{H}=0,18 \mathrm{~m} \\
& \mathrm{~B}=0,252 \mathrm{~m}
\end{aligned}
$$

- Velocidad horizontal ( $\left.\mathrm{V}_{\mathrm{H}}\right)$ : (Arboleda, 2000) recomienda que la velocidad horizontal o de arrastre debe ser menor a $0,55 \mathrm{~cm} / \mathrm{s}$.

$$
\begin{gathered}
V_{H}=\frac{Q}{B H} \\
V_{H}=\frac{9,25 \times 10^{-6} \mathrm{~m}^{3} / \mathrm{s}}{0,2572 \mathrm{~m} \times 0.18 \mathrm{~m}} \\
V_{H}=0,01998 \mathrm{~cm} / \mathrm{s}
\end{gathered}
$$


Donde:

$\mathrm{VH}=$ Velocidad horizontal

$\mathrm{Q}=$ Caudal $\left(\mathrm{m}^{3}\right)$

$\mathrm{B}=$ Ancho $(\mathrm{m})$

$\mathrm{H}=$ Profundidad $(\mathrm{m})$

b. Zona de Entrada: En esta zona se debe cumplir con los siguientes criterios:

- Gradiente $\leq 20 \mathrm{~s}^{-1}$ o el gradiente de la última cámara.

- Velocidad de paso $(0,1-0,3) \mathrm{m} / \mathrm{s}$.

- Ubicación de orificios superiores $(1 / 5$ - 1/6) H desde la superficie del agua. Los inferiores de $(1 / 4-1 / 5) \mathrm{H}$.

- Pared o cortina difusora se ubicará a $(0,7-1) \mathrm{m}$ a partir de la pared externa del sedimentador.

- Área total de orificio (ATO)

$$
\begin{gathered}
A T_{O}=\frac{Q_{D}}{V_{P}} \\
A T_{O}=\frac{9,25 \times 10^{-6} \mathrm{~m}^{3} / \mathrm{s}}{4,90 \times 10^{-3} \mathrm{~m} / \mathrm{s}} \\
A T_{O}=0,00189 \mathrm{~m}^{2}
\end{gathered}
$$

- Número o cantidad de orificios (No) Se consideraron 24 orificios en la placa de $25,72 \mathrm{~cm} \mathrm{x} 18 \mathrm{~cm}$

El diámetro de orificios debe ser asumido respecto al siguiente intervalo $(3-6)$ in. Se tomará un diámetro de 4 in $(0,01 \mathrm{~m})$.

- Área de cada orificio (Ao)

$$
\begin{aligned}
& A_{O}=\frac{\pi D^{2}}{4}=\frac{\pi(0,01)^{2}}{4} \\
& A_{O}=7,85 \times 10^{-5} \mathrm{~m}^{2}
\end{aligned}
$$

- Área total de orificios (ATo)

$$
\begin{gathered}
N_{O}=\frac{A T_{O}}{A_{O}} \\
A T_{O}=N_{O} \times A_{O}=24 \times 7,85 \times 10^{-5} \mathrm{~m}^{2} \\
A T_{O}=188 \times 10^{-5} \mathrm{~m}^{2}
\end{gathered}
$$

- Velocidad de paso $\left(\mathrm{V}_{\mathrm{P}}\right)$

$$
\begin{gathered}
A T_{O}=\frac{Q_{D}}{V_{P}} \\
V_{P}=\frac{Q_{D}}{A T_{O}}=\frac{9,25 \times 10^{-6} \mathrm{~m}^{3} / \mathrm{s}}{188,4 \times 10^{-5} \mathrm{~m}^{2}} \\
V_{P}=4,90 \times 10^{-3} \mathrm{~m} / \mathrm{s}
\end{gathered}
$$




\section{Diseño del sedimentador laminar}

a. Dimensionamiento de las tuberías de distribución

- Caudal (Q): Se realizó la prueba a un caudal determinado.

$\mathrm{Q}=9,25 \mathrm{~mL} / \mathrm{s}$

$\mathrm{Q}=9,25 \times 10^{-6} \mathrm{~m}^{3} / \mathrm{s}$

- Tubería de distribución de flujo de 1,08 m con 12 orificios en cada tubería de 1 $\mathrm{cm}$ de diámetro con espaciado proporcional.

- Cálculo del área del orificio (A0)

$$
\begin{gathered}
A_{0}=\frac{\pi D^{2}}{4}=\frac{\pi(0,01)^{2}}{4} \\
A_{0}=7,85 \times 10^{-5} \mathrm{~m}^{2}
\end{gathered}
$$

- Área total de orificios (AT0)

$$
\begin{gathered}
N_{0}=\frac{A T_{0}}{A_{0}} \\
A T_{O}=N_{0} \times A_{0}=24 \times 7,85 \times 10^{-5} \mathrm{~m}^{2} \\
A T_{O}=188,4 \times 10^{-5} \mathrm{~m}^{2}
\end{gathered}
$$

Donde:

$\mathrm{N}_{0}=$ Número de orificios.

$\mathrm{AT}_{0}=$ Área total de orificios.

$\mathrm{A}_{0}=$ Área de cada orificio.

- $\quad$ Velocidad de paso (VP)

$$
\begin{gathered}
A T_{0}=\frac{Q_{D}}{V_{P}} \\
V_{p}=\frac{9,25 \times 10^{-6} \mathrm{~m}^{3} / \mathrm{s}}{188,4 \times 10^{-5} \mathrm{~m}^{2}} \\
V_{p}=4,90 \times 10^{-3} \mathrm{~m} / \mathrm{s}
\end{gathered}
$$

b. Dimensionamiento del sedimentador laminar

- Datos: Se realizó una separación entre placas (e') de 0,05 m. El espesor de las placas (e) fue de $6 \mathrm{~mm}$, y el ángulo de inclinación ( $(\theta)$ fue de $60^{\circ}$.

- Cálculo del espaciamiento entre placas (d)

$$
\begin{gathered}
\mathrm{d}=e^{\prime} x \sin \theta-e \\
\mathrm{~d}=0,05 m \times \sin 60^{\circ}-0,006 m \\
\mathrm{~d}=0,0373 m
\end{gathered}
$$

- Longitud del módulo de placas (L)

$\mathrm{L}=0,06 \mathrm{~m}$

Cálculo de la longitud útil (Lu)

$$
\begin{gathered}
L u=L-e^{\prime} x \cos \theta=0,06-0,05\left(\cos 60^{\circ}\right) \\
L u=0,0350 m
\end{gathered}
$$


Cálculo de la longitud relativa del módulo de placas (L)

$$
\begin{aligned}
L=\frac{L u}{d} & =\frac{0,0350 m}{0,0373 m} \\
L & =0,94
\end{aligned}
$$

- Cálculo del coeficiente del módulo de placas $(f)$

Sea la eficiencia (S): $\mathrm{S}=1$

La velocidad de sedimentación de partículas (VS)

$$
\begin{gathered}
V_{S}=\frac{Q}{A_{S}}=\frac{9,25 \times 10^{-6} \mathrm{~m}^{3} / \mathrm{s}}{1,08 \mathrm{~m} \times 0,26 \mathrm{~m}} \\
V_{S}=3,29 \times 10^{-5} \mathrm{~m} / \mathrm{s}
\end{gathered}
$$

Donde:

$$
\begin{aligned}
& \mathrm{Q}=\text { Caudal }\left(\mathrm{m}^{3} / \mathrm{s}\right) \\
& \mathrm{A}_{\mathrm{S}}=\text { Área superficial }\left(\mathrm{m}^{2}\right)
\end{aligned}
$$

El coeficiente del módulo de placas $(f)$ :

$$
\begin{gathered}
f=\left[\sin 60^{\circ}\left(\sin 60^{\circ}+0.94 x \cos 60^{\circ}\right)\right] \\
f=1.15
\end{gathered}
$$

- Cálculo del área superficial corregida de la unidad (ASc)

$$
\begin{gathered}
A_{S c}=\frac{Q}{V_{S} \times f}=\frac{9,25 \times 10^{-6} \mathrm{~m}^{3} / \mathrm{s}}{3,29 \times 10^{-5} \mathrm{~m} / \mathrm{s} \times 1,15} \\
A_{S c}=0,244 \mathrm{~m}^{2}
\end{gathered}
$$

- Cálculo del número de canales formados por las placas $(\mathrm{N})$

$$
\begin{aligned}
N=\frac{A_{S c} \times \sin \theta}{B \times d} & =\frac{0,244 \mathrm{~m}^{2} \times \sin 60^{\circ}}{0,26 m \times 0,0373 \mathrm{~m}} \\
N & =21,789
\end{aligned}
$$

Donde:

$\mathrm{As}_{\mathrm{S}}=$ Área superficial de la unidad $\left(\mathrm{m}^{2}\right)$

$\mathrm{B}=$ Ancho neto de la zona de decantación $(\mathrm{m})$

$\mathrm{d}=$ Distancia entre placas $(\mathrm{m})$

- Cálculo de la longitud total del decantador (LT)

$$
\begin{gathered}
L_{T}=\frac{\{L \cos \theta+[N d+(N+1) e]\}}{\sin \theta} \\
L_{T}=\frac{0,06 x \cos 60^{\circ}+[22(0,0373)+(22+1) x 0,006]}{\sin 60^{\circ}} \\
L_{T}=1,14 \mathrm{~m}
\end{gathered}
$$


- Cálculo de la velocidad media del flujo (Vo)

$$
\begin{gathered}
V_{O}=\frac{Q}{A_{S C} \times \sin \theta}=\frac{9,25 \times 10^{-6} \mathrm{~m}^{3} / \mathrm{s}}{0,244 \mathrm{~m}^{2} \times \sin 60^{\circ}} \\
V_{O}=4,38 \times 10^{-5} \mathrm{~m} / \mathrm{s}
\end{gathered}
$$

- Cálculo del radio hidráulico $(\mathrm{RH})$

Siendo el ancho $\mathrm{B}=0,26$

$$
\begin{gathered}
R_{H}=\frac{B \times d}{2(B+d)}=\frac{0,26 \times 0,0373}{2(0,26+0,0373)} \\
R_{H}=0,0163 \mathrm{~m}
\end{gathered}
$$

- Cálculo del número de Reynolds (NR)

Sea la viscosidad a $25^{\circ} \mathrm{C}=8,9 \times 10^{-3} \mathrm{~cm}^{2} / \mathrm{s}$

$$
\begin{gathered}
N R=4 R_{H} \times \frac{V_{O}}{V}=6,52 \mathrm{~cm} \times \frac{4,38 \times 10^{-3} \mathrm{~cm} / \mathrm{s}}{8,9 \times 10^{-3} \mathrm{~cm}^{2} / \mathrm{s}} \\
N R=3,21
\end{gathered}
$$

Dimensiones del sedimentador laminar:

$$
\begin{aligned}
& \text { L: } 1,08 \mathrm{~m} \\
& \text { B: } 0,26 \mathrm{~m} \\
& \text { H: } 0,18 \mathrm{~m}
\end{aligned}
$$

Canaleta de lodos:

$$
\begin{aligned}
& \text { HT: } 0,18+0,1(1,08) \\
& \text { HT: } 0,28 \\
& : 0,05 \mathrm{~m}
\end{aligned}
$$

Ancho de la canaleta

Bordes del agua de salida : 0,05 m

Espesor de vidrio: $6 \mathrm{~mm} \quad: 0,006 \mathrm{~m}$

Test de jarras: Primero se preparó agua con tierra a 1, 2 y $3 \%$ de concentración y la dosis de floculantes el test de jarras (tabla1)

Tabla 1. Test de jarras para determinar la dosis de floculante

\begin{tabular}{ccccc}
\hline Concentración & \multicolumn{2}{c}{ Sedimentador Convencional } & \multicolumn{2}{c}{ Sedimentador Laminar } \\
\cline { 2 - 5 }$(\mathbf{\%})$ & Dosis & Caudal & Dosis & Caudal \\
& óptima $(\mathrm{mL})$ & $(\mathrm{mL} / \mathrm{min})$ & óptima $(\mathrm{mL})$ & $(\mathrm{mL} / \mathrm{min})$ \\
$\mathbf{1}$ & 8 & 29,60 & 25 & 46,25 \\
$\mathbf{2}$ & 70 & 129,50 & 75 & 138,75 \\
$\mathbf{3}$ & 75 & 135,75 & 75 & 138,75 \\
\hline
\end{tabular}




\section{Puesta en marcha de los sedimentadores}

Se consideró las recomendaciones de la CEPIS ${ }^{5}$. Para ello se agregó el agua preparada con las diferentes concentraciones de sólidos a un caudal de $9,25 \mathrm{~mL} / \mathrm{s}$, y se tomaron los datos a la entrada y salida de los sedimentadores. Esta primera etapa fue sin coagulante, después que pasó el agua se colectó y se volvió a pasar en los sedimentadores utilizando coagulante.

\section{Determinación de parámetros fisicoquímicos}

Determinación de turbiedad del agua. Se preparó una solución de cloruro férrico al $10 \%$, se realizó un barrido en el espectrofotómetro UV Visible en un rango de 400 a $600 \mathrm{~nm}$ de longitud de onda que presenta máxima absorción. Luego se prepararon soluciones estándares de 2, 6, 10, 14 y 18 ppm para levantar la curva estándar, una vez levantada la curva estándar se tomó lectura de las muestras problema de los sedimentadores, los resultados se expresaron como partes por millón (ppm) equivalentes en cloruro férrico.

Determinación del pH del agua. Se realizó empleando el método electrométrico. El método consiste en la determinación de la actividad de los iones hidrógeno por medidas potenciométricas usando un electrodo combinado o un electrodo estándar de hidrógeno de vidrio con un electrodo de referencia.

Determinación del OD del agua. Se utilizó el método de Winkler o yodométrico.

Determinación de la temperatura del agua: La temperatura del agua se midió con un termómetro digital.

Determinación del contenido de sólidos totales del agua (residuo seco). Se pesaron los vasos precipitados vacíos, luego se tomaron $20 \mathrm{ml}$ de agua residual de concentraciones de sólidos al 1,2 y $3 \%$, dichas muestras se llevaron a una cocina eléctrica a $100{ }^{\circ} \mathrm{C}$ por un aproximado de 30 minutos, donde se evaporaron las $3 / 4$ partes del total del volumen, seguidamente se llevaron las muestras a una estufa a $103{ }^{\circ} \mathrm{C}$ por 12 horas aproximadamente, luego se llevaron al desecador para el enfriamiento de las muestras, y se pesaron los vasos precipitados con los sólidos sedimentados en ellos. Para la determinación de residuo seco se realizó el siguiente cálculo: (W: Peso g)

$$
\text { RESIDUO SECO }=W_{\text {VASO CON SÓLIDOS }}-\boldsymbol{W}_{\text {VASO VACIO }} \quad \text { Eq } 1
$$

\section{Evaluación de la eficiencia de los sedimentadores}

$$
\begin{array}{ll}
\text { EFICIENCIA }=\left(\frac{\boldsymbol{E}-\boldsymbol{S}}{\boldsymbol{E}}\right) \boldsymbol{x} \mathbf{1 0 0} \% & \text { Eq } 2 \\
\text { E: } & \text { Turbiedad del agua a la entrada del sedimentador (ppm) } \\
\text { S: } & \text { Turbiedad del agua a la salida del agua del sedimentador (ppm) }
\end{array}
$$

La eficiencia se determinó para Residuos Secos obtenidos de las muestras de agua a la entrada y salida del sedimentador. 
Análisis estadístico. Los datos se analizaron bajo el diseño de bloques completamente al azar (DCA), considerándose el análisis de varianza y correlación de Tukey, con un arreglo factorial de 2Ax2Bx3C, con un Modelo Aditivo Lineal. Se utilizó el software estadístico R.

\section{RESULTADOS Y DISCUSIÓN}

\section{Turbiedad}

Las concentraciones de sólidos de $2 \%$ y $3 \%$ presentan mayor disminución de sólidos que la concentración en $1 \%$. El sedimentador laminar obtuvo mayor disminución de turbiedad $(45,278 \mathrm{ppm})$ en comparación del convencional (32,545 ppm) (figura 1) y las concentraciones de sólidos de $2 \%$ y $3 \%$ generaron mayor disminución de sólidos que la concentración de $1 \%$ (figura 1). La eliminación de lodos en los sedimentadores se produce bajo condiciones especiales como el tiempo de retención, diseño de los sedimentadores, oxígeno disuelto, $\mathrm{pH}$ y otros factores ${ }^{6}$. Los criterios antes mencionados son importantes para reducir la turbidez del agua, otro factor importante es la velocidad de flujo, se encontró que de 1 a $3 \mathrm{mms}^{-1}$ producen mayor rendimiento de flóculos en turbiedades ente 10 y 200 NTU, mientras una velocidad de flujo ascendente entre 0,6 y $0,8 \mathrm{mms}^{-1}$ produce mayor rendimiento de flóculos para turbiedades de $500 \mathrm{NTU}^{7}$, según los resultados encontrados la velocidad de flujo utilizada fue óptima para la concentración inicial de sólidos de $2 \%$ (figura 1). En el presente estudio la velocidad horizontal fue $0,02 \mathrm{~cm} / \mathrm{s}$ para el sedimentador convencional y $0,04 \mathrm{~cm} / \mathrm{s}$ para el sedimentador laminar, se siguió los criterios de la CEPIS para no tener problemas de arrastre. Se obtuvo mayor remoción de sólidos en el sedimentador laminar que en el convencional (figura 1).

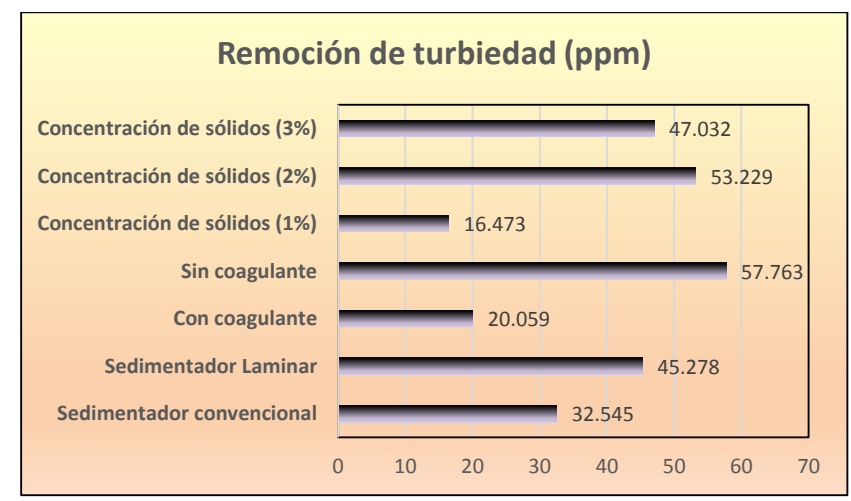

Figura 1. Remoción de turbiedad en los sedimentados con y sin coagulante en diferentes concentraciones de sólidos

\section{Coagulante:}

La remoción de sólidos depende de la composición química del agua, cuando los sólidos están disueltos generalmente se usa floculantes. Para sales de aluminio el rango de $\mathrm{pH}$ para la coagulación es de 6,5 a 8,0 y para las sales de hierro, el rango de pH óptimo es de 5,5 a 8,5 unidades asimismo la cantidad del coagulante a utilizar tiene influencia directa en la eficiencia de la coagulación4, coincide con el presente estudio que se utilizó floculante de 
aluminio y se ajustó el $\mathrm{pH}$ alrededor de 6,3 y se obtuvo como resultado una buena cantidad de disminución de la turbiedad $(57,763 \mathrm{ppm})$ con la concentración inicial de sólidos de $2 \%$ (figura 1). Sin coagulante se obtuvo mayor remoción de turbiedad, esto se debe a que la mayoría de sólidos estaban en suspensión y el peso específico era alto y podían precipitarse solos, asimismo el coagulante se usó después que se eliminó los sólidos en suspensión, se utilizó para los sólidos disueltos siendo necesario para clarificar el agua. Los procesos de coagulación floculación dependen de los contaminantes. El tratamiento de agua residual con productos intermedios de foto degradación de diclofenaco se obtuvo una remoción de $60 \%$ de sólidos después de la filtración de Uv- Cloración, pero no después de coagulación/ floculación ${ }^{8}$, se aprecia que la naturaleza de sólidos disueltos del agua influye en el uso de los coagulantes y los procedimientos a utilizar, la remoción de sólidos depende de la naturaleza de los mismos, del tipo de floculante y otros factores que se determinan en cada proceso específico. Asimismo, es importante innovar tecnologías, el desarrollo de floculadores que incluyen sistema de eliminación de lodos en el instante, evita el desbordamiento y el menor tiempo de residencia de los lodos ${ }^{9}$, lo que conlleva a reducir tiempos y optimizar procesos.

\section{Oxígeno disuelto $(O D)$}

El uso del coagulante presentó el oxígeno disuelto, presentando mayor variación del oxígeno disuelto utilizando el coagulante que sin coagulante, entre la salida y entrada de los sedimentadores (figura 2). Asimismo, el oxígeno disuelto aumenta en el sedimentador laminar (figura 2), el tratamiento con mayor incremento del oxígeno disuelto es con coagulante en la concentración de sólidos de $2 \%$. En las concentraciones $1 \%$ y $2 \%$ generó mayor variación de OD, más aún en la concentración de $2 \%$ se obtuvo más variación, mientras que fue diferente al uso de la concentración en $3 \%$. La presencia de oxígeno disuelto en el agua cruda depende de la temperatura, la presión y la mineralización del agua, los sólidos disueltos, carga orgánica y otros. La ley de Henry y Dalton dice: "La solubilidad de un gas en un líquido es directamente proporcional a la presión parcial e inversamente proporcional a la temperatura", se determinó que existe mayor variación en el aumento de oxígeno disuelto en el sedimentador laminar donde presentó mayor remoción de turbidez (figura 1) y a la vez en las concentraciones de 2 y $3 \%$ (figura 2). El incremento del oxígeno disuelto en el sedimentador laminar fue mayor que en el convencional.

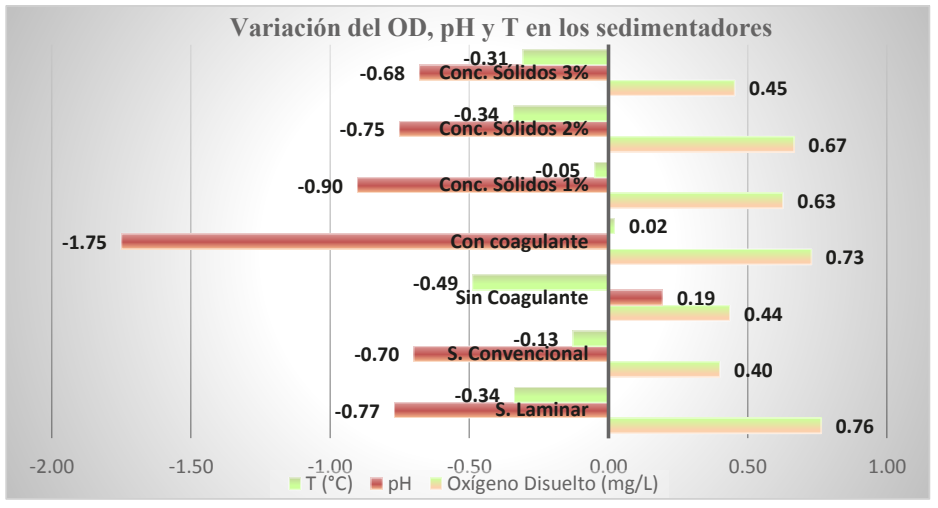

Figura 2. Variación de OD, pH y temperatura en los sedimentadores 
Residuo seco (sólidos totales)

El uso del coagulante incrementó la remoción de sólidos totales, aumentando la variación del residuo seco que sin usar coagulante (figura 3). Las concentraciones $2 \%$ y $3 \%$ generó mayor variación en el residuo seco siendo diferente en la concentración de $1 \%$ (figura 3). El residuo seco en el sedimentador laminar presentó una mayor variación que en el sedimentador convencional (figura 3), y los tratamientos que presentaron mayor disminución del residuo seco fue con coagulante (figura 3). La aplicación de una dosis creciente del coagulante en el agua presenta diferentes zonas de coagulación ${ }^{4}$. Esta afirmación se puede corroborar en los resultados obtenidos de residuo seco (figura 3) y turbiedad (figura 1), para concentraciones de sólidos de $1 \%$ la disminución de residuo seco y turbiedad es deficiente que para las concentraciones con $2 \%$ la disminución se incrementa en comparación a las aguas con concentraciones de $3 \%$, se corrobora que la concentración que presenta mayor disminución de turbiedad es del $3 \%$ (figura 2). Se obtuvo mayor disminución de sólidos en el sedimentador laminar que en el convencional

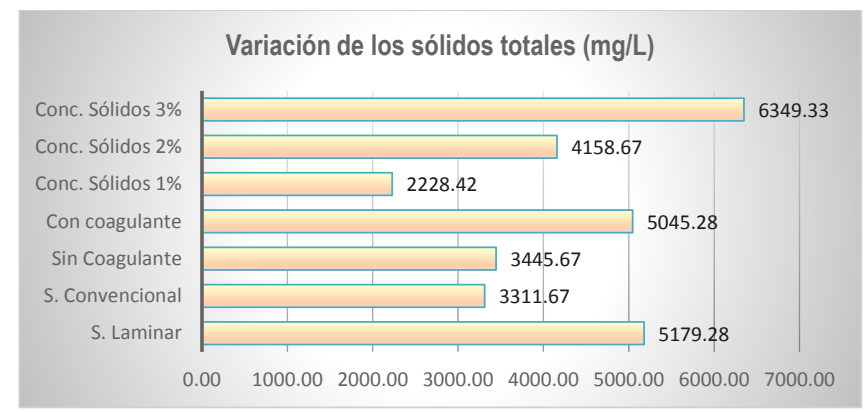

Figura 3. Variación de los sólidos totales en la entrada y salida de los sedimentadores.

\section{Evaluación de la eficiencia de los sedimentadores}

La eficiencia de un sedimentador debe ser como mínimo $85 \%$ en la separación de sólidos sedimentables. Para esto es necesario verificar que la trayectoria de cada partícula tenga como destino el fondo del sedimentador. Uno de los procesos que más afecta la eficiencia de los sedimentadores es la remoción de la turbidez del fluido y en la calidad del agua obtenida, es el proceso de coagulación y floculación, ya que una adecuada selección del coagulante y una eficiente dosificación del mismo genera una mayor cantidad de formación de partículas grandes en el floculador, para posteriormente ser eliminadas en el sedimentador ${ }^{3}$. Los resultados obtenidos en el sedimentador laminar corroboran con lo antes mencionado ya que se obtuvo un rendimiento de 93,5\% y en el convencional de $78 \%$ (figura 4), en este trabajo se evaluó distintas concentraciones de sólidos con resultados diferentes sugiriendo que los resultados de eficiencia de los sedimentadores dependen de la turbiedad del agua y las características de los sólidos presentes. Se encontró que los sedimentadores laminares soportan de mejor manera el aumento de la carga superficial que los sedimentadores convencionales ${ }^{3}$. También se reporta que los recipientes de sedimentación con paredes inclinadas paralelas son capaces de aclarar el agua más rápidamente que los convencionales, debido a su mayor área superficial ${ }^{10}$, se demostró que el sedimentador laminar es más eficiente; 
esto se debe a que los sedimentadores laminares presentan placas inclinadas que incentivan a la precipitación de los sólidos suspendidos y de los flóculos. El clarificador jet puede reducir efectivamente la turbidez del agua con una eficiencia del $80 \%$ bajo condiciones óptimas de coagulación y floculación ${ }^{11}$, como se aprecia la eficiencia de los sedimentadores tiene mucha influencia en las características del diseño, así como otros factores. Los métodos de diseño novedosas, basadas en el comportamiento dinámico de flujo y la distribución de la densidad en clarificadores, se necesitan con el fin de mejorar la eficacia de sistemas de tratamiento de aguas residual ${ }^{12}$. El diseño utilizado en el sedimentador laminar con las placas inclinadas contribuyó notablemente en la remoción de la turbidez del agua. El movimiento impulsado por la gravedad de las partículas rígidas en un fluido viscoso es relevante en muchos procesos naturales e industriales ${ }^{13}$. El complejo proceso dinámico de sedimentación, clarificación y lodos activos, son sensibles a cambios en la carga hidráulica orgánica ${ }^{14}$. Finalmente, la eliminación de sólidos disueltos y suspendidos en aguas residuales son un riesgo para la salud, debido a que es un foco infeccioso de contagio de Norovirus humano, la calidad del agua, se puede mejorar en gran parte con los tratamientos primarios y lodos activados ${ }^{15}$.

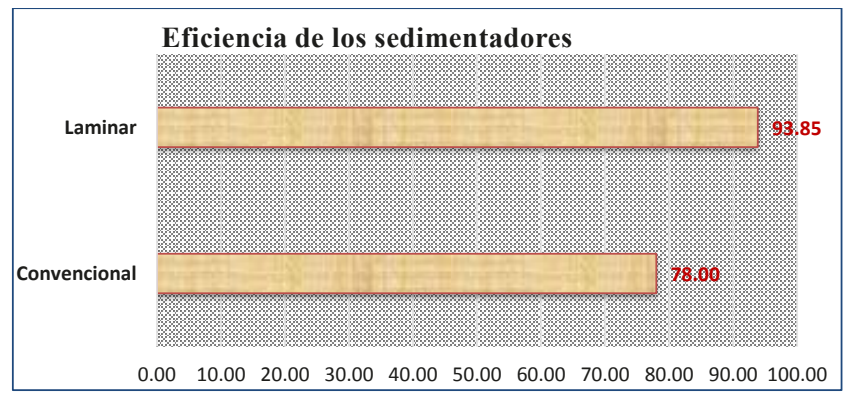

Figura 4. Eficiencia de los sedimentadores.

\section{CONCLUSIONES}

- El sedimentador laminar presentó mayor disminución en la turbiedad $(45,278 \pm 2,897$ ppm) que el convencional (32,545 $\pm 2,897 \mathrm{ppm})$. Asimismo, se removió 3311,67 y 5179,28 de sólidos totales para el sedimentador convencional y laminar respectivamente.

- El sedimentador laminar presentó mayor incremento de oxígeno disuelto en 0,764 \pm $0,076 \mathrm{mg} / \mathrm{L}$ que el convencional $0,400 \pm 0,076 \mathrm{mg} / \mathrm{L}$

- El sedimentador laminar (93,85\%) es más eficiente en comparación al sedimentador convencional $(78,00 \%)$, teniendo a la concentración del $2 \%$ como la más efectiva y con tratamiento con coagulante.

- La eficiencia de un sedimentador se ve influenciado por las características de diseño y los parámetros de operación. 


\section{REFERENCIAS BIBLIOGRÁFICAS}

1. Malcolm B., Johnson M., Elphinston A, Ratnayaka D. Twort's Water Supply. Oxford. Elsevier. 2017. $933 \mathrm{p}$

2. Trujillo D., Duque L., Aralla J, Rincón A. Pacheco S, Herrera O. Remoción de Turbiedad

3. Huerta R., Vélez E. Implementación de un sedimentador laminar de flujo horizontal a escala reducida en el Laboratorio Agroindustrial de la ESPAM-MFL. Escuela Superior Politécnica Agropecuaria de Manabí Manuel Félix López. Calceta, Ecuador. 2012. 136 p.

4. Andía, Y. Tratamiento de Agua: Coagulación y Floculación. SEDAPAL. $1^{\circ}$ Edición. Lima, Perú. 2000. 47 p

5. CEPIS, OPS. Guía para el diseño de desarenadores y sedimentadores. Organización Panamericana para la Salud. Lima, Perú. 2005.34 p

6. Bertanza G., Pedrazzani R., Manili L. Laura Menoni a Bio-P release in the final clarifiers of a large WWTP with co-precipitation: Key factors and troubleshooting Chemical Engineering Journal 230. 2013. 195-201

7. Hurst M., Weber-Shirk M., Leonard W. Lion Parameters affecting steady-state floc blanket performance Journal of Water Supply: Research and Technology AQUA. 2010. 59.5

8. Matamoros V., Salvadó V. Evaluation of a coagulation/flocculation-lamellar clarifier and filtration-UV-chlorination reactor for removing emerging contaminants at full-scale wastewater treatment plants in Spain Journal of Environmental Management. España. 117 (2013) 96102

9. Alastair D. Optimization of clarifier-thickeners processing stable suspensions for turnup/turn-down Water Research 38 (2004) 1568-1578

10. Romphophak P., Wongwailikhit K., Chawaloesphonsiya N., Samornkraisorakit P., Painmanakul P. Study of flow pattern in jet clarifier for removal of turbidity by residence time distribution approach Engineering Journal Volume 20, Issue 2, 18 May 2016, Pages $17-27$

11. Davis R., Gecol H. Classification of concentrated suspensions using inclined settlers Int. J. Multiphase Flow. 1996. Vol. 22, No. 3, pp. 563-574,

12. Deininger A., Giinthert F., Wilderer P. The influence of currents on circular secondary clarifier performance and design Waf. Sci. Tech. 1996. Vol. 34, No. 3-4, pp. 405-412

13. Ardekani M., Costa P., Breugem P., Brandt L. Numerical study of the sedimentation of spheroidal particles International Journal of Multiphase Flow 87 (2016) 16-34

14. Koehne M., Hoen K., Schuhen, M. Modelling and simulation of final clarifiers in wastewater treatment plants Mathematics and Computers in Simulation 39 (1995) 609616

15. Campos C., Avant J., Lowther J., Till D., Lees D. Human norovirus in untreated sewage and effluents from primary, secondary and tertiary treatment processes Water Research 103 (2016) 224e232 (20) 